\title{
Do standard carotid artery endarterectomy and primary closure technique cause early restenosis in diabetic patients?
}

Eyup Murat Kanber ${ }^{1,2}$, Murat Ugurlucan ${ }^{1}$, Mazlum Sahin $^{1}$, Devrim Saribal ${ }^{3}$, Ufuk Alpagut ${ }^{1}$

\author{
${ }^{1}$ Department of Cardiovascular Surgery, Medical Faculty, Istanbul University, Istanbul, \\ Turkey \\ ${ }^{2}$ Istanbul Education and Research Hospital, Ministry of Health, Istanbul, Turkey \\ ${ }^{3}$ Department of Biophysics, Medical Faculty, Istanbul University, Istanbul Cerrahpaşa, \\ Turkey
}

Submitted: 3 October 2017

Accepted: 16 November 2017

Arch Med Sci Atheroscler Dis 2017; 2: e103-e107

DOI: https://doi.org/10.5114/amsad.2017.72534

Copyright @ 2017 Termedia \& Banach

\begin{abstract}
Introduction: We aimed to investigate carotid restenosis in 2-year follow-up in diabetic and nondiabetic patients who underwent standard carotid endarterectomy with primary carotid closure and determine whether diabetes mellitus is a risk factor for early stenosis for this surgical procedure.

Material and methods: We retrospectively assessed the data of patients who underwent standard carotid endarterectomy with primary carotid closure from the hospital registry and outpatient clinic follow-up between January 2006 and January 2012. The study included 25 diabetics and 25 nondiabetics, in total 50 patients. The control carotid Doppler ultrasonographies and/or computed tomography angiographies of the patients at postoperative $1,6,12$, and 24 months were examined and a stenosis rate between $70 \%$ and $99 \%$ was regarded as significant carotid restenosis.

Results: When the diabetic and nondiabetic group patients were compared for early carotid restenosis at 2 years, there were $3(12 \%)$ patients in the diabetic group and $4(16 \%)$ patients in the nondiabetic group with restenosis. A statistically significant difference in early carotid restenosis was not observed between the two groups $(p>0.05)$.

Conclusions: Standard carotid endarterectomy and primary closure of the artery is a successfully performed surgical procedure in diabetic patients. We concluded that diabetes mellitus is not a risk factor for early restenosis in the diabetic patient population according to the results of our research.
\end{abstract}

Key words: carotid artery stenosis, diabetes mellitus, endarterectomy.

\section{Introduction}

Carotid endarterectomy (CEA) is current and effective treatment applied in the treatment of symptomatic and asymptomatic carotid artery stenosis in the current era. Carotid artery restenosis developing after carotid endarterectomy is an important health issue [1]. Several studies have demonstrated the carotid artery restenosis rate in the range of 6-36\% [2-6]. The main reason for restenosis to have such a wide range is that the carotid endarterectomy techniques applied in the studies, techniques for arteriotomy closure and clinical characteristics of patients differ widely. Methods such as primary closure of arteriotomy, closure

\author{
Corresponding author: \\ Dr. Eyup Murat Kanber \\ Ministry of Health \\ Istanbul Education \\ and Research Hospital \\ Cardiovascular Surgery Clinic \\ Istanbul, Turkey \\ Phone: +905057952530 \\ Fax: +902125342232 \\ E-mail: eyupmuratkanber@ \\ yahoo.com
}


with a venous patch, closure with a Dacron patch, closure with a PTFE patch, eversion endarterectomy and standard endarterectomy are commonly used surgical techniques [7]. Studies comparing all these surgical techniques in terms of restenosis development after the operation and involving large series of patients are available in the literature. In addition to the evidence related to reduction in restenosis development with patches in many randomized trials, there are also studies showing that the same results were obtained with primary closure.

The clinical features of the patients are as important as the surgical technique performed in the development of restenosis after carotid endarterectomy [2]. While there are studies indicating that hyperlipidemia, diabetes and female gender affect the development of restenosis after CEA, there are also studies suggesting the opposite [3, 4].

In this study, we aimed to compare diabetic and nondiabetic patients in terms of early carotid artery restenosis development after standard CEA and primary closure and to examine whether this surgical procedure and diabetes mellitus cause an additional increase in risk for the development of early carotid artery restenosis.

\section{Material and methods}

In this study, the hospital records of the patients who underwent a standard CEA operation in our department and in whom the arteriotomy was primarily closed between January 2006 and January 2012 were examined, retrospectively. A total of 50 patients including randomly selected 25 nondiabetic and 25 diabetic patients who came to the follow-up visits regularly at $1,6,12$ and 24 months were included in the study. The diabetic patients were insulin-dependent and/or using an oral antidiabetic. Patients in whom conventional CEA was performed but the arteriotomy was closed with a patch, those in whom other techniques such as eversion CEA was performed, those operated on due to restenosis and those who underwent carotid endarterectomy concurrent with coronary artery bypass operation were excluded from the study.

The investigations of control carotid Doppler ultrasonography (USG) and/or carotid computed tomography (CT) angiography performed during the postoperative 1, 6, 12 and 24 months follow-up visits of the patients were examined and the data were recorded. The additional clinical features of the patients which may affect the development of carotid artery restenosis such as age, gender, smoking, hypertension, hyperlipidemia and preoperative carotid artery stenosis degree in percentage were recorded. In the preoperative period, $100 \mathrm{mg}$ acetylsalicylic acid (ASA) and $20 \mathrm{mg}$ atorvastatin were initiated. Low molecular weight heparin at the anticoagulant dose $(0.1 \mathrm{mg} / \mathrm{kg})$ was added to the treatment of the patients in the postoperative period for $48 \mathrm{~h}$. At discharge, clopidogrel $75 \mathrm{mg}$ along with ASA was added to the treatment of the patients and dual antiaggregant therapy was continued for 1 year. Afterwards, ASA therapy was continued lifelong. Detection of $70 \%$ or more carotid artery stenosis in any of the control carotid Doppler USG and/or carotid CT angiography performed during any of the follow-up visits at 1, 6, 12 and 24 months was considered as carotid artery restenosis.

\section{Surgical procedure}

The operations were performed with infiltration anesthesia with a $50 \%$ mixture of prilocaine hydrochloride and bupivacaine hydrochloride local injection. If the patient complained of pain or discomfort, repeated doses were injected. A standard incision parallel to the sternocleidomastoid muscle was performed and the common carotid artery (CCA), internal carotid artery (ICA), and external carotid artery (ECA) were prepared and dissected. After systemic 5000 IU of heparin injection, the arteries were clamped. The consciousness and the neurologic status of the patient were evaluated with his or her response to verbal stimuli and ability to move the contralateral side hand and foot for at least 3 min prior to the arteriotomy. In case of neurologic disturbance, endarterectomy was performed with insertion of a shunt. The carotid endarterectomy and primary repair was performed in the usual fashion.

\section{Statistical analysis}

Statistical analysis was performed using the Statistical Package for Social Sciences 15.0 program (SPSS for Windows, SPSS Inc, Chicago, IL). The variables were expressed as mean \pm standard deviation. Nonparametric methods were used in the statistical analysis of the data. The variables obtained at different times were compared using the Mann-Whitney $U$ test. Repeated values were analyzed by ANOVA test. The differences between two groups were assessed by Kruskal-Wallis analysis of variance and Student's $t$ test. The correlation analysis of the data was performed with Spearman rank correlation analysis. Categorical variables were compared using the $\chi^{2}$ test. The results were evaluated at the confidence interval of $95 \%$ and significance level of $p<0.05$.

\section{Results}

The patients included in the study were named as the diabetic group and nondiabetic group. Of the patients in the diabetic group, 6 were female 
and 19 were male and their mean age was 62.2 \pm 7 years. Of the patients in the nondiabetic group, 8 were female and 17 were male and their mean age was $65 \pm 12.7$ years. In the diabetic group, 15 patients had hypertension and 8 patients had hyperlipidemia, while in the nondiabetic group, 12 patients had hypertension and 3 patients had hyperlipidemia. Active smoking history was present in 3 patients of the diabetic group and in 5 patients of the nondiabetic group. The mean preoperative carotid artery stenosis rate was calculated to be $80.2 \pm 9.5 \%$ for the patients in the diabetic group and as $82 \pm 10.3 \%$ for the patients in the nondiabetic group. No statistically significant difference was detected in terms of clinical characteristics such as gender, age, hypertension, hyperlipidemia and preoperative carotid artery stenosis rate which could affect carotid artery restenosis in both groups of patients. The demographic data of the patients are shown in Table I.

No mortality was observed in the patients. No cerebral neurological event occurred in any patient in the peroperative and postoperative periods. Partial asymmetry of the face due to platismal nerve injury was accepted as a local neurological event and it was observed in 5 patients in both groups. Mean operation duration was 55 min. All patients were transfered to the ward after being monitored for $24 \mathrm{~h}$ in the intensive care unit and they were discharged from the hospital after an average of $72 \mathrm{~h}$. The total follow-up period was 36 months.

Comparing the patients with and without diabetes in terms of restenosis, it was observed in $3(12 \%)$ patients of the diabetic group and in $4(16 \%)$ patients of the nondiabetic group. No neurological event associated with restenosis occurred in any patient. There was no statistically significant difference between the two groups in terms of nerurological events. The patients with restenosis were treated with stent implantation. The postoperative data of the patients are shown in Table II.

When the patients with restenosis were grouped as diabetic and nondiabetic, the mean age of the nondiabetic patient group was $65 \pm 2$ years and the mean age of the diabetic group was $64 \pm 1$ years. Of the diabetic patients in whom restenosis developed 1 was female, and in the nondiabetic group 2 were female. There was no statistically significant difference between the two groups in terms of hypertension, hyperlipidemia or smoking history. The mean preoperative carotid artery stenosis value of the diabetic group patients was $76.6 \pm 5 \%$ while it was $81.25 \pm 7 \%$ for the nondiabetic group. The data are presented in Table III.

\section{Discussion}

One of the major problems after carotid endarterectomy is early or late restenosis development [1]. Although most of the patients in whom restenosis develops remain asymptomatic, they are more likely to have cerebrovascular events than those in whom restenosis does not develop [1]. The main goal of the treatment in patients with carotid artery stenosis is to prevent current or future cerebrovascular diseases. Thus prevention of restenosis after CEA is an important part of the treatment in patients with carotid artery stenosis [1].

The development of restenosis after carotid endarterectomy is considered in two periods [2].

Table I. Comparison of clinical features of nondiabetic and diabetic groups

\begin{tabular}{|lccc|}
\hline Parameter & $\begin{array}{c}\text { Diabetic group } \\
(n=25)\end{array}$ & $\begin{array}{c}\text { Nondiabetic group } \\
(n=25)\end{array}$ & $P$-value \\
\hline Age [years] & $62.24 \pm 7$ & $65 \pm 12.7$ & 0.442 \\
\hline Women & $6(24 \%)$ & $8(32 \%)$ & 0.529 \\
\hline Men & $19(76 \%)$ & $17(68 \%)$ & 0.529 \\
\hline Hypertension (+) & $15(60 \%)$ & $12(48 \%)$ & 0.395 \\
\hline Hyperlipidemia (+) & $8(32 \%)$ & $3(12 \%)$ & 0.088 \\
\hline Preoperative carotid artery stenosis (\%) & $80.2 \pm 9.5$ & $82 \pm 10.3$ & 0.297 \\
\hline
\end{tabular}

Table II. Comparison of nondiabetic and diabetic groups in terms of restonosis

\begin{tabular}{|lcc|}
\hline Parameter & Restenosis (+) & Restenosis (-) \\
\hline Nondiabetic group $(n=25)$ & $4(16 \%)$ & $21(84 \%)$ \\
\hline Diabetic group $(n=25)$ & $3(12 \%)$ & $22(88 \%)$ \\
\hline Total $(n=50)$ & $7(14 \%)$ & $43(86 \%)$ \\
\hline
\end{tabular}


Table III. Comparison of demographic data of nondiabetic restenosis (+) and diabetic restenosis (+) groups

\begin{tabular}{|lcc|}
\hline Parameter & Diabetic restenosis $(+),(n=3)$ & Nondiabetic restenosis $(+),(n=4)$ \\
\hline Age [years] & $64 \pm 1$ & $65 \pm 2$ \\
\hline Women & $1(33 \%)$ & $2(50 \%)$ \\
\hline Men & $2(67 \%)$ & $2(50 \%)$ \\
\hline Hypertension (+) & $1(33 \%)$ & $1(25 \%)$ \\
\hline Smoking history & $1(33 \%)$ & $1(25 \%)$ \\
\hline Hyperlipidemia (+) & 0 & 0 \\
\hline Preoperative carotid artery stenosis (\%) & $76.60 \pm 5$ & $81.25 \pm 7$ \\
\hline
\end{tabular}

The first period is the early carotid artery restenosis which develops within the first 2 years after the operation and which is suggested to be due to intimal hyperplasia. The second period is the late carotid artery restenosis which develops after the second year and the etiology of which is suggested to be atherosclerosis [2].

One of the most important questions for the patients in whom carotid artery surgery planned due to carotid artery stenosis is 'Which surgical technique should be used?' Standard CEA and primary closure is a well established technique among the other surgical techniques. The shorter cross-clamp time of this technique compared to other techniques is an advantage accepted by many authors [8].

One of the questions arising when evaluating carotid artery restenosis is which stenosis rate will be considered as significant carotid artery restenosis. There are many studies accepting different restenosis rates, such as $50 \%, 60 \%$, or $70 \%$, as significant. In our study, we accepted the stenosis rate between the range of $70 \%$ and $99 \%$ to which carotid artery restenosis is recommended whether it is symptomatic or not as significant carotid artery restenosis.

Carotid artery restenosis has been the subject of many studies in the past. As there are studies conducted only on surgical techniques (standard CEA, eversion endarterectomy, closure with venous, Dacron, or PTFE patch, etc.) without taking the clinical characteristics of the patients into account, there are also studies considering only the clinical characteristics (age, gender, hypertension, diabetes mellitus, hyperlipidemia, etc.) and neglecting surgical techniques. In this study, we applied a certain surgical technique in two groups with similar characteristics except diabetes and thereby we tried to minimize the effects of other clinical characteristics suggested to affect restenosis rates.

In the study by Rerkasem and Rothwell on 1967 patients, they obtained limited evidence that closure with the patch angioplasty technique reduced the risk of restenosis compared to primary closure [9]. Reinert and Mono suggested that the rate of restenosis was very low after a standard carotid artery endarterectomy operation performed under a microscope without using patches in their study including 586 patients [8]. In the study by Beşirli et al. evaluating 20 patients who underwent CEA, they recommended the addition of patch angioplasty to endarterectomy in order to reduce the risk of premature thrombosis and late restenosis [10]. In our study, the primary closure technique was applied to all patients. Considering all patients, the rate of restenosis was found to be $14 \%$ (50 patients, 7 restenosis). This rate is in parallel with previous studies on this subject.

The gender and clinical characteristics of the patients such as diabetes, hyperlipidemia and hypertension have also been the subjects of many studies. Cossman et al. observed an early restenosis rate of $3.6 \%$ in their study including 361 patients. They determined that hypertension and hyperlipidemia were more frequent in the patients with restenosis [5]. In the study by Brau et al. in which 43 patients underwent CEA and were followed up for 3 years, the rate of restenosis between 70 and $99 \%$ was found to be $4.3 \%$. They determined the major risk factors for restenosis as hypertension, hyperlipidemia, alcohol and smoking [11]. Reina-Gutiérrez et al. identified the risk factors for restenosis as female gender and diabetes in their retrospective study including 243 CEA operations performed on 224 patients [12]. In the study by Kogure et al. including 135 CEA operations performed on 126 patients, they did not detect any statistically significant factor for carotid artery restenosis except female gender [13]. Cuming et al. followed up a total of 107 patients including 71 males and 36 females for 1 year. The number of patients who developed more than $50 \%$ restenosis was found to be 18 (17\%). They found that restenosis was not associated with age, hypertension, diabetes or gender [14]. In the study by Liu et al. including 572 patients, they compared 184 diabetic patients 
with nondiabetic patients and did not detect a statistically significant difference between the two groups in terms of carotid artery restenosis (diabetic $2.9 \%$ and nondiabetic $3.4 \%$ ) compared to nondiabetic patients [15]. In the study Mizuhashi et al. including 281 patients, they compared 136 diabetic patients with the remaining nondiabetic patients and found no significant difference between the two groups in terms of carotid artery restenosis [16]. As can be understood from these examples, there are conflicting results from the previous studies on carotid artery restenosis.

A major limitation of the study is the small size of the cohort, which included 50 patients divided into two groups as the diabetic and nondiabetic patients. However, the current study presents the preliminary results of our research. The cohort is expanding with patients admitted to our clinic as well as the data from other cardiovascular surgery centers, and the results will be submitted as a supplement to the same journal if something crucial is found. Another limitation is the retrospective nature of the research.

In conclusion, the results of our study indicate that early restenosis rates after carotid endarterectomy in diabetic and nondiabetic patients were similar when taking into account identical groups with similar cardiovascular risk factors, which is consistent with previous studies in the literature suggesting that diabetes is not a risk factor for early restenosis. However, with only 50 patients, the study is vastly under-powered to address the research question at hand. Multicenter, high patient volume studies are warranted in order to reach a definite conclusion on the issue.

\section{Acknowledgments}

The study includes results of the residency thesis of Eyup Murat Kanber, MD.

Eyup Murat Kanber, MD conveys sincere gratitude to Prof. Turkan Tansel for her remarkable help.

\section{Conflict of interest}

The authors declare no conflict of interest.

\section{References}

1. Reichmann BL, de Borst GJ, Moll FL. How to avoid restenosis after carotid endarterectomy. Presented at the $38^{\text {th }}$ Annual Vascular and Endovascular Issues, Techniques and Horizons (VEITH symposium, 2011 November 16-20).

2. Kan P, Mokin M, Dumont TM, Snyder KV, Siddiqui AH. Cervical carotid artery stenosis: latest update on diagnosis and management. Curr Probl Cardiol 2012; 37: 127-69.

3. Schanzer A, Hoel A. Restenosis after carotid endarterectomy performed with routine intraoperative Duplex ultrasonography and arterial patch closure: a contemporary series. Vasc Endovascular Surg 2007; 41: 200-5.

4. Benavente O, Moher D, Pham B. Carotid endarterectomy for asymptomatic carotid stenosis: a meta-analysis. BMJ 1998; 317: 1477-80.

5. Cossman D, Allan D, Avrum Stein DO, Matsumoto G. Early restenosis after carotid endarterectomy. Arch Surg 1978; 113: 275-8.

6. Thomas M, Otis SM, Rush M, Zyroff J, Dilley RB, Bernstein EF. Recurrent carotid artery stenosis following endarterectomy. Ann Surg 1984; 200: 74-9.

7. Golledge J, Cuming R, Davies AH, Greenhalgh RM. Outcome of selective pachting following carotid endarterctomy. Eur J Vasc Endovasc Surg 1996; 11: 458-63.

8. Reinert M, Mono ML. Restenosis after microsurgical non-patch carotid endarterectomy in 586 patients. Acta Neurochir 2012; 154: 423-31.

9. Rerkasem K, Rothwell PM. Patch angioplasty versus primary closure for carotid endarterectomy. Cochrane Database Syst Rev 2009; 4: CD000160.

10. Beşirli K, Bozkurt K, Kaynak K, et al. Karotid Endarterektomileri (20 olgu Analizi). GKD Cer Derg 1994; 1-2: 148-51.

11. Brau RH, Betancourt AJ, Vásquez R, Brau RR, Colberg R. Carotid artery restenosis in a Hispanic population. Health Sci J 2008; 27: 315-21.

12. Reina-GutiérrezT,Serrano-HernandoFJ,Sánchez-HervásL, Ponce A, Vega de Ceniga M, Martín A. Recurrent carotid artery stenosis following endarterectomy: natural history and risk factors. Eur J Vasc Endovasc Surg 2005; 29: 334-41.

13. Kogure S, Sakai N, Murao K, et al. [Restenosis after CEA: pathogenesis and treatment]. No Shinkei Geka 2002; 30: 1303-12.

14. Cuming R, Worrell P, Woolcock NE, Franks PJ, Greenhalgh RM, Powell JT. The influence of smoking and lipids on restenosis after carotid endarterectomy. Eur J Vasc Surg 1993; 7: 572-6.

15. Liu XN, Ni L, Wang WD, Zeng R, Liu CW. Perioperative safety analysis of carotid endarterectomy in diabetic patients. Zhonghua Yi Xue Za Zhi 2016; 96: 3549-53.

16. Mizuhashi S, Kataoka H, Sano N, et al. Impact of diabetes mellitus on characteristics of carotid plaques and outcomes after carotid endarterectomy. Acta Neurochir (Wien) 2014; 156: 927-33. 